\title{
On the Attractor of the Product System
}

\author{
Guifeng Huang ${ }^{1}$ and Lidong Wang ${ }^{2}$ \\ ${ }^{1}$ Department of Basic Science, Dalian Naval Academy, Dalian, Liaoning 116018, China \\ ${ }^{2}$ Department of Mathematics, Dalian Nationalities University, Dalian, Liaoning 116600, China
}

Correspondence should be addressed to Lidong Wang; wld@dlnu.edu.cn

Received 1 June 2013; Accepted 7 August 2013

Academic Editors: G. Stavroulakis and W. Yeih

Copyright (C) 2013 G. Huang and L. Wang. This is an open access article distributed under the Creative Commons Attribution License, which permits unrestricted use, distribution, and reproduction in any medium, provided the original work is properly cited.

We investigate the attractor of the product system and mainly prove that the likely limit set of the product system equals the product of one of each factor system for $n$ compact systems with solenoid attractors. Specially, this holds for the product map of Feigenbaum maps. Furthermore, we deduce that the Hausdorff dimension of the likely limit set of the product map for Feigenbaum maps is the sum of one of each factor map.

\section{Introduction}

An attractor plays a very important role in the studies of dynamical system. The concept of the likely limit set was introduced by Milnor in 1985 (see [1]). As indicated in [1], the likely limit set always exists. Because this kind of attractors gathers the asymptotic behaviors of almost all points, it is very necessary to study them.

In [2], an analytic function similar to a unimodal Feigenbaum map (we call the solution of Feigenbaum functional equation Feigenbaum map) was investigated; the Hausdorff dimension of the likely limit set of it was estimated. We discussed the dynamical properties of unimodal Feigenbaum map, estimated the Hausdorff dimension of the likely limit set for the unimodal Feigenbaum map, and proved that for every $s \in(0,1)$, there always exists a unimodal Feigenbaum map such that the Hausdorff dimension of the likely limit set is $s$. We also considered the kneading sequences of unimodal Feigenbaum maps (see [3]). Similarly to this, we studied the nonunimodal Feigenbaum maps in [4].

In this paper, we explore the dynamics of the product map whose every factor map has solenoid attractor and show that, for this kind of product map, the likely limit set has multiplicative property; that is, the likely limit set of the product map equals the product of one of each factor system. As an application, we consider the product of Feigenbaum maps. The main results are Theorems 8 and 10.

\section{Basic Definitions and Preparations}

Milnor introduced the concept of the likely limit set in 1985 $[1]$.

Definition 1. Let $M$ be a compact manifold (with boundary possibly) and $f$ a continuous map of $M$ into itself. The likely limit set $\Lambda=\Lambda(f)$ of $f$ is the smallest closed subset of $M$ with the property that $\omega(x, f) \subset \Lambda$ for every point $x \in M$ outside of a set of Lebesgue measure zero $(\omega(x, f)$ denotes the $\omega$-limit set of the point $x$ under $f$ ).

A set $E \subset I$ is called a minimal set of $f$ if $E \neq \phi$ and $\omega(x, f)=E$ for every point $x \in E$. As is well known, the minimal set is a nonempty, closed, and invariant subset under $f$, and it has no proper subset with these three properties (see [5]). Therefore, if $E$ is a minimal set with $\omega(x, f) \subset E$ for almost all $x \in I$, then $E=\Lambda(f)$.

The notion of period interval is an extension of one of period point [6].

Definition 2. Let $I$ be a real compact interval and $C(I)$ the set of continuous maps from $I$ into itself. Let $N$ be the set of positive integers, $f \in C(I)$. A sequence $\left(I_{k}\right)_{k=0}^{n-1}$ of closed subintervals of $I$ is said to be periodic of $n$ if they have disjoint interiors and $f\left(I_{k}\right) \subset I_{k+1}$ for any $k=0,1, \ldots, n-2$ and $f\left(I_{n-1}\right) \subset I_{0}$. Call $C=\bigcup_{k=0}^{m-1} I_{k}$ a cycle of periodic intervals. 
A C $I$ is called a solenoid of $f$ if there are a strictly increasing sequence $\left(k_{n}\right)_{n=1}^{\infty}$ of positive integers and periodic sequences $\left(I_{k}^{n}\right)_{k=0}^{k_{n}-1}$ of closed intervals of period $k_{n}$ such that $\bigcup_{k=0}^{k_{n}-1} I_{k}^{n} \supset \bigcup_{k=0}^{k_{n+1}-1} I_{k}^{n+1}$ for any $n$ and $A=\bigcap_{n=1}^{\infty} \bigcup_{k=0}^{k_{n}-1} I_{k}^{n}$.

We call the family $\left\{\left(I_{k}^{n}\right)_{k=0}^{k_{n}-1}\right\}_{n=1}^{\infty}$ a covering of $A$ of type $\left(k_{n}\right)_{n=1}^{\infty}$. If a solenoid admits a covering of type $\left(2^{n}\right)_{n=1}^{\infty}$, we call it a doubling period solenoid of $f$.

Let $X, Y$ be $m, n$ dimensional compact space, respectively. Descartes product of $X$ and $Y$ is $X \times Y=\left\{(x, y) \in R^{m+n} \mid x \in\right.$ $X, y \in Y\} ; x * y$ denotes Descartes product of $x$ and $y$.

Let $f: X \rightarrow X, g: Y \rightarrow Y$ be continuous maps. For all $(x, y) \in X \times Y$,

$$
(f \times g)(x, y)=(f(x), g(y)) .
$$

The concepts of $\omega$-limit set and likely limit set in one dimension can be extended to the product space.

In 1978, Feigenbaum [7] put forward Feigenbaum functional equation:

$$
\begin{gathered}
g^{2}(-\lambda x)=-\lambda g(x), \\
g(0)=1, \quad-1 \leq g(x) \leq 1, \quad x \in[-1,1],
\end{gathered}
$$

where $\lambda \in(0,1)$ is to be determined.

In 1985, Yang and Zhang [8] proposed the second type of Feigenbaum functional equation:

$$
\begin{gathered}
f^{2}(\lambda x)=\lambda f(x), \\
f(0)=1, \quad 0 \leq f(x) \leq 1, \quad x \in[0,1],
\end{gathered}
$$

where $\lambda \in(0,1)$ is to be determined.

There is a close link between the solutions of these two types of equations.

Lemma 3. Let $g(x)$ be a unimodal Feigenbaum map. If, for $x \in[\lambda, g(\lambda)], g^{\prime}(x)<-1$, and, for $x \in[g(\lambda), 1], g^{\prime}(x) \leq-1$ (considering the left or right derivative at the end points), then

(1) the likely limit set $\Lambda(g)$ is a minimal set of $g$;

(2) $s \leq \operatorname{dim} \Lambda(g) \leq t$,

where

$$
\begin{aligned}
\lambda^{s}(1+ & \left.\left(\inf _{x \in[\mu, 1]} g^{\prime}(x)\right)\right)^{-s} \\
& =1=\lambda^{t}\left(1+\left(\sup _{x \in[\mu, 1]} g^{\prime}(x)\right)\right)^{-t},
\end{aligned}
$$

and $\operatorname{dim}(\cdot)$ denotes the Hausdorf dimension.

For a proof see [3].

Lemma 4. For everys $\in(0,1)$, there exists a unimodal Feigenbaum map g such that $\operatorname{dim} \Lambda(g)=s$.

For a proof see [3].
Lemma 5. Let $f(x)$ be a nonunimodal Feigenbaum map and $\mu$ the minimum point of $f$ on $[\lambda, 1]$. If, for $x \in[\lambda, \mu], f^{\prime}(x)<-1$ and, for $x \in[\mu, 1], f^{\prime}(x) \geq 1$ (considering the left or right derivative at the end points), then

(1) there exists a set of contractions such that its invariant set is the likely limit set $\Lambda(f)$ and a minimal set of $f$;

(2) $s \leq \operatorname{dim} \Lambda(f) \leq t$,

where

$$
\begin{aligned}
\lambda^{s}(1+ & \left.\left(\inf _{x \in[\mu, 1]} f^{\prime}(x)\right)\right)^{-s} \\
& =1=\lambda^{t}\left(1+\left(\sup _{x \in[\mu, 1]} f^{\prime}(x)\right)\right)^{-t},
\end{aligned}
$$

and $\operatorname{dim}(\cdot)$ denotes the Hausdorff dimension.

For a proof see [4].

Lemma 6. For every $s \in(0,1)$, there always exists a nonunimodal Feigenbaum map such that $\operatorname{dim} \Lambda(f)=s$.

For a proof see [4].

Lemma 7. If E, $F$ are well-distributed Cantor sets, then

$$
\operatorname{dim}(E \times F)=\operatorname{dim} E+\operatorname{dim} F,
$$

where $\operatorname{dim}(\cdot)$ denotes the Hausdorff dimension.

For a proof see [9].

\section{The Theorems and Their Proofs}

Theorem 8 (main theorem). Let $I$, J be compact intervals, $S=$ $I \times J$, and let $f: I \rightarrow I, g: J \rightarrow J$ be continuous, and let $\Lambda(f), \Lambda(g)$ be solenoid attractors; then for $f \times g: S \rightarrow S$, one has

$$
\Lambda(f \times g)=\Lambda(f) \times \Lambda(g) .
$$

Proof. Let $f, g$ be as indicated in the theorem.

Consider

$$
\begin{aligned}
& \Lambda(f)=\bigcap_{n=1}^{\infty} \bigcup_{p=0}^{p_{n}-1} I_{p}^{n}, \\
& \Lambda(g)=\bigcap_{n=1}^{\infty} \bigcup_{q=0}^{q_{n}-1} J_{q}^{n},
\end{aligned}
$$

where $\left\{p_{n}\right\}_{n=1}^{\infty},\left\{q_{n}\right\}_{n=1}^{\infty}$ are strictly increasing sequences of positive integer; $\left\{I_{p}^{n}\right\}_{p=0}^{p_{n}-1},\left\{J_{q}^{n}\right\}_{q=0}^{q_{n}-1}$ are two sequences of closed intervals with period $p_{n}$ and $q_{n}$, respectively.

First, we show $\Lambda(f \times g) \subset \Lambda(f) \times \Lambda(g)$.

By the definition of likely limit set, we assume that $\omega(x, f) \subset \Lambda(f)$ for every $x \in I$ outside of the set $I_{0} \subset I$ with Lebesgue measure zero and $\omega(y, g) \subset \Lambda(g)$ for every 
$y \in J$ outside of the set $J_{0} \subset J$ with Lebesgue measure zero. Since $\Lambda(f)$ and $\Lambda(g)$ are closed sets, $\Lambda(f) \times \Lambda(g)$ is a compact invariant set; then it is a closed set. $\Lambda(f \times g)$ is the smallest invariant closed set that attracts almost all points in $S$. Thus, it is enough to prove that $\Lambda(f) \times \Lambda(g)$ attracts almost all points in $S$ in order to show $\Lambda(f \times g) \subset \Lambda(f) \times \Lambda(g)$.

Because any $x \in I-I_{0}, \omega(x, f) \subset \Lambda(f)$, any $y \in J-J_{0}$, $\omega(y, g) \subset \Lambda(g)$, for any $(x, y) \in I \times J-\left(I_{0} \times J\right) \cup\left(I \times J_{0}\right)$, it is easy to know

$$
\begin{aligned}
\omega(x * y, f \times g) & \subset \omega(x, f) \times \omega(y, g) \\
& \subset \Lambda(f) \times \Lambda(g) .
\end{aligned}
$$

Since $\left(I_{0} \times J\right) \cup\left(I \times J_{0}\right)$ is a Lebesgue measure zero set, it follows that $\Lambda(f) \times \Lambda(g)$ attracts almost all points in $S$. Therefore,

$$
\Lambda(f \times g) \subset \Lambda(f) \times \Lambda(g)
$$

Second, we prove that $\Lambda(f) \times \Lambda(g) \subset \Lambda(f \times g)$.

Suppose that there is a point $\left(x_{0}, y_{0}\right) \in \Lambda(f) \times \Lambda(g)$, but $\left(x_{0}, y_{0}\right) \bar{\epsilon} \Lambda(f \times g) . \Lambda(f \times g)$ is a closed set, so there exists an open neighborhood $U \times V \subset S$ such that $\left(x_{0}, y_{0}\right) \in U \times V$ and $(U \times V) \cap \Lambda(f \times g)=\phi$.

By the definition of solenoid attractor, there must be period intervals $I_{p_{0}} \subset I, J_{q_{0}} \subset J$ containing $x_{0}, y_{0}$, respectively. Assume that the period of $I_{p_{0}}$ is $m_{1}$, the one of $J_{q_{0}}$ is $m_{2}$; then the one of $I_{p_{0}} \times J_{q_{0}}$ is not more than $m$, where $m=m_{1} \times m_{2}$. So for any $(x, y) \in I_{p_{0}} \times J_{q_{0}}$,

$$
\begin{aligned}
(f \times g)^{m}(x * y) & \in(f \times g)^{m}\left(I_{p_{0}} \times J_{q_{0}}\right) \\
& \subset I_{p_{0}} \times J_{q_{0}} \subset U \times V,
\end{aligned}
$$

$I_{p_{0}} \times J_{q_{0}}$ has positive Lebesgue measure, this shows the neighborhood $U \times V$ of $\left(x_{0}, y_{0}\right)$ attracts a positive measure set, contrary to the definition of $\Lambda(f \times g)$. Therefore, this assumption does not hold. So

$$
\Lambda(f) \times \Lambda(g) \subset \Lambda(f \times g)
$$

Sum up, we obtain

$$
\Lambda(f \times g)=\Lambda(f) \times \Lambda(g)
$$

Obviously, this conclusion holds for finite maps.

Corollary 9. Let $f_{i}: I_{i} \rightarrow I_{i}$ be a continuous map, where $I_{i}$ is a compact interval and $\Lambda\left(f_{i}\right)$ is a solenoid attractor, $i=$ $1,2, \ldots, m$; then

$$
\begin{aligned}
\Lambda\left(f_{1} \times\right. & \left.f_{2} \times \cdots \times f_{m}\right) \\
& =\Lambda\left(f_{1}\right) \times \Lambda\left(f_{2}\right) \times \cdots \times \Lambda\left(f_{m}\right) .
\end{aligned}
$$

Specially, this holds for the unimodal Feigenbaum map and the nonunimodal Feigenbaum map which we have discussed in $[3,4]$, respectively.
Theorem 10. Let $I, J=[0,1]$ or $[-1,1]$; let $f: I \rightarrow I, g$ : $J \rightarrow J$ be the unimodal Feigenbaum map or the nonunimodal Feigenbaum map as in Lemma 3 or Lemma 5, $f \times g: I \times J \rightarrow$ $I \times J$; then

$$
\Lambda(f \times g)=\Lambda(f) \times \Lambda(g)
$$

Proof. Let $J=[-1,1]$. First, we show $\Lambda(g)$ is a doubling period solenoid of $g$ if $g$ is a unimodal Feigenbaum map of $J$ into itself. Let $\bar{U}=[-\lambda, 1]$.

By the proof of Lemma 3 (see [3]), we know

$$
\begin{aligned}
\Lambda(g) & =\bigcap_{k=0}^{\infty} \varphi^{k}(\bar{U}) \\
& =\bigcap_{k=0}^{\infty} \bigcup_{i_{1}, \ldots, i_{k}=1}^{2} \varphi_{i_{1}, \ldots, i_{k}}(\bar{U}) .
\end{aligned}
$$

For any $\varphi_{i_{1}, \ldots, i_{k}}(\bar{U}), \varphi_{j_{1}, \ldots, j_{k}}(I)$, there exists $n>0$ such that $g^{n} \circ$ $\varphi_{i_{1}, \ldots, i_{k}}(\bar{U})=\varphi_{j_{1}, \ldots, j_{k}}(\bar{U})$; then

$$
\varphi^{k}(\bar{U})=\bigcup_{m=0}^{2^{k}-1} g^{m} \circ \varphi_{i_{1}, \ldots, i_{k}}(\bar{U}) .
$$

It is easy to see that

$$
g^{2^{k}-1} \circ \varphi_{i_{1}, \ldots, i_{k}}(\bar{U})=\varphi_{i_{1}, \ldots, i_{k}},
$$

and $\varphi^{k}(\bar{U})$ is the sum of $2^{k}$ closed sets which are disjoint from each other; that is, $\varphi^{k}(\bar{U})$ is a sequence of closed intervals with period $2^{k} \cdot \varphi^{k+1}(\bar{U})$ is obtained by removing an open interval in each connected component of $\varphi^{k}(\bar{U})$ and $\bar{U} \supset \varphi(\bar{U}) \supset \cdots \supset$ $\varphi^{k}(\bar{U}) \supset \cdots$. So $\Lambda(g)$ is a doubling period solenoid of $g$.

Similarly to this, we can also show that $\Lambda(f)$ is a doubling period solenoid of $f$ if $f$ is a nonunimodal Feigenbaum map of $[0,1]$ into itself.

Hence, by Theorem 8 we get

$$
\Lambda(f \times g)=\Lambda(f) \times \Lambda(g) .
$$

Theorem 11. If $f, g$ are the unimodal Feigenbaum maps as in Lemma 3 or the nonunimodal Feigenbaum maps as in Lemma 5, then

$$
\operatorname{dim} \Lambda(f \times g)=\operatorname{dim} \Lambda(f)+\operatorname{dim} \Lambda(g) .
$$

Proof. It is easy to know from Theorem 10 and Lemma 7.

By Lemmas 4 and 6 and Theorem 11, we immediately have the following.

Corollary 12. For any $t \in(0,2)$, there are Feigenbaum maps $f, g$ such that

$$
\operatorname{dim} \Lambda(f \times g)=t
$$




\section{Conclusion}

We discussed the likely limit set of product system, show that the likely limit set of product map of two maps with solenoid likely limit sets equals the product of one of each factor map, apply this to the unimodal Feigenbaum map and the nonunimodal Feigenbaum map, and know that the Hausdorff dimension of the likely limit set of the product map of two Feigenbaum maps is the sum of one of each factor map.

\section{Acknowledgment}

The authors wish to thank the NSFC (no. 11271061) for financial support.

\section{References}

[1] J. Milnor, "On the concept of attractor," Communications in Mathematical Physics, vol. 99, no. 2, pp. 177-195, 1985.

[2] K. J. Falconer, The Geometry of Fractal Sets, vol. 85, Cambridge University Press, Cambridge, UK, 1986.

[3] G. F. Huang, L. D. Wang, and G. F. Liao, "A note on the unimodal Feigenbum's maps," International Journal of Modern Physics B, vol. 14, pp. 3101-3111, 2009.

[4] G. F. Huang and L. D. Wang, "On the attractors of Feigenbaum maps," Annales Polonici Mathematici. In press.

[5] L. S. Block and W. A. Coppel, Dynamics in One Dimension, vol. 1513 of Lecture Notes in Mathematics, Springer, Berlin, Germany, 1992.

[6] V. J. López and L. Snoha, "There are no piecewise linear maps of type $2^{\infty}$," Transactions of the American Mathematical Society, vol. 349, no. 4, pp. 1377-1387, 1997.

[7] M. J. Feigenbaum, "Quantitative universality for a class of nonlinear transformations," Journal of Statistical Physics, vol. 19, no. 1, pp. 25-52, 1978.

[8] L. Yang and J. Z. Zhang, "The second type of Feigenbaum's functional equations," Science China, vol. 12, pp. 1061-1069, 1985 (Chinese).

[9] K. Falconer, Fractal Geometry: Mathematical Foundations and Applications, John Wiley \& Sons, Chichester, UK, 1990. 


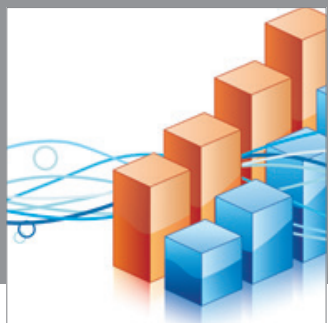

Advances in

Operations Research

mansans

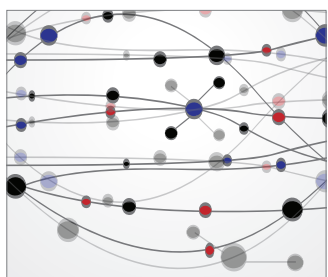

The Scientific World Journal
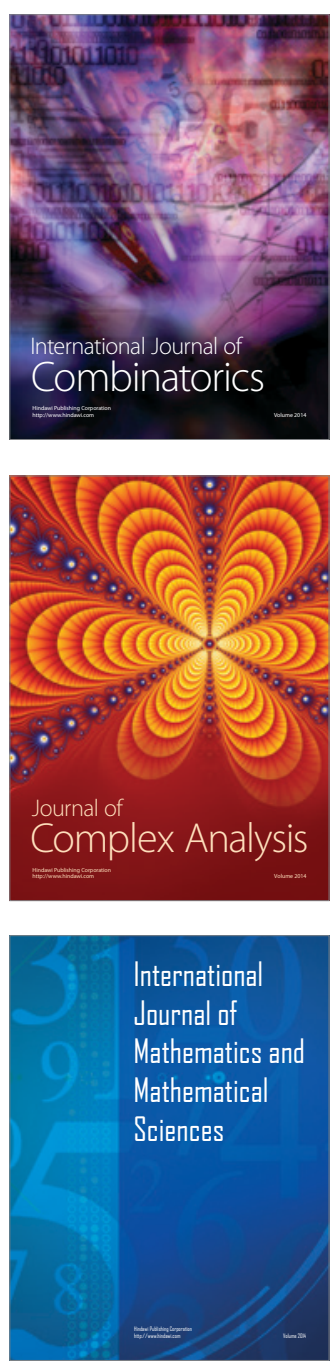


Submit your manuscripts at http://www.hindawi.com


Journal of

Function Spaces

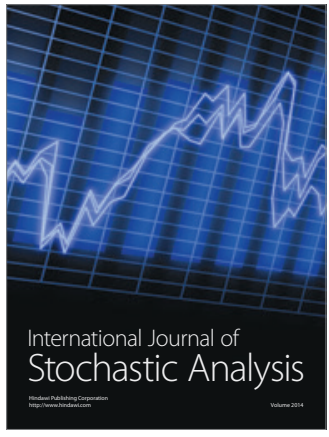

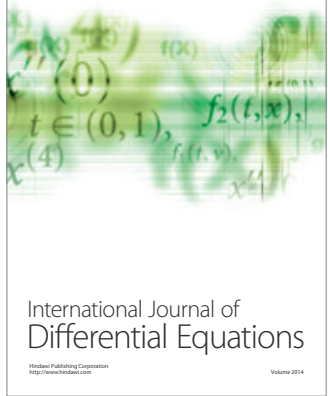
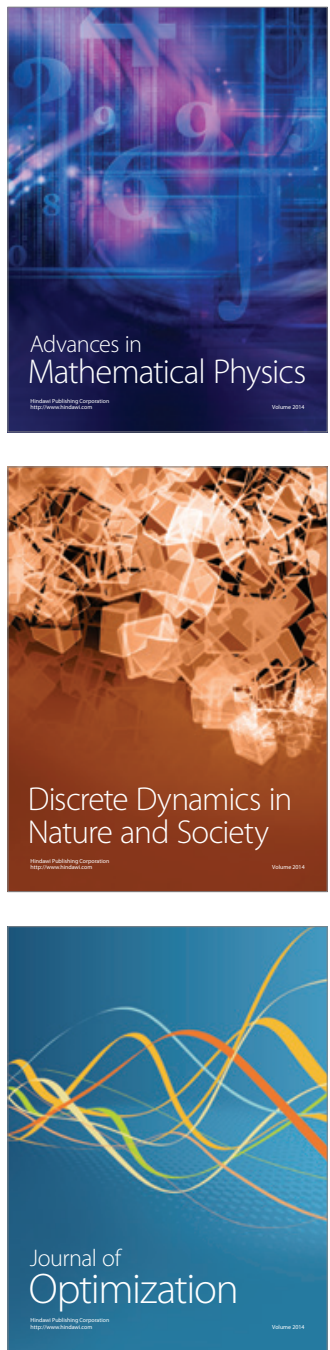